\title{
A Framework for Evaluating Collaborative Product Representations in Product Lifecycle Workflows
}

\author{
Nathan Hartman ${ }^{1}$, Phil Rosche ${ }^{2}$, and Kevin Fischer ${ }^{3}$ \\ ${ }^{1}$ Purdue University, West Lafayette, IN USA \\ nhartman@purdue.edu \\ ${ }^{2}$ ICF International, Charleston, SC USA \\ proscheaicfi.com \\ ${ }^{3}$ Rockwell Collins, Cedar Rapids, IA USA \\ klfische@rockwellcollins.com
}

\begin{abstract}
Product definition technology has evolved in such a way as to encourage the proliferation of data formats used within the product lifecycle for various use cases. One such product development framework being proposed in industry today is the model-based enterprise (MBE), which is operationally driven by the model-based definition (MBD) of products. This paper describes current models of the product development process and the nature of collaborative data. This paper will also suggest a framework for evaluating various collaborative product representations. The nature of the data formats chosen for use in specific workflows have a substantial effect on the ability of users to consume data and the ability for an organization to capture and archive its critical information.
\end{abstract}

Keywords: product lifecycle management, model-based enterprise, modelbased definition, neutral file formats, workflows, standards.

\section{Introduction}

The modern product realization environment, and the accompanying product lifecycle, has expanded in such a fashion that the global design, production, distribution, and support of a product is commonplace. Technologies and techniques, such as crowd sourcing, mobile devices, and social networks, have led to new ways that people communicate product design information. The notion of collaborative design and development activities have become commonplace due to the evolution of computing and communications technology and the globalization of productproducing companies [1,2]. In an effort to effectively do business within this environment, companies have turned to using 3D Computer Aided Design (CAD)based product representations as the basis for their communication [3,1]. In doing so, engineers are able to capture product definitions with a higher degree of fidelity and a reduced risk of error based on interpretation of design intent. However, it is not to say that the use of 3D models is not without risk. According to an Aberdeen study [4], 269 companies were surveyed, and $84 \%$ of them reported using three or more CAD 
tools in their design process. Forty-two percent of the respondents reported using five or more CAD systems. Why all of the variety? The number one reason cited by respondents was that product development is not done in isolation - they needed to collaborate with other people in other organizations who ultimately used different tools and processes. As different digital product representation capabilities have developed within modern CAD authoring software, they can be classified as data models, data formats, and data wrappers (or packaging). This paper will provide a discussion of the nuances of using these different product representations in the product development processes, and a framework for evaluating these digital product representations in typical product lifecycle workflows.

With the proliferation of CAD software, and the ongoing incompatibilities between native $\mathrm{CAD}$ tools, the communication process between participants in the product lifecycle can be treacherous. Collaboration between people can take many forms and can use many different types of data and information formats. In the collaborative product design and PLM spaces, this data will typically take one of three forms relative to the aforementioned use of CAD-based product definitions to enable communication between members of a team: native files, neutral files, or lightweight files. Native files are those created and stored in the format of the CAD authoring tool (e.g., CAD, PDM, spreadsheet, word processing, presentation, logistics, etc.) Neutral formats are broadly interpretable file formats that can be used between different applications (e.g., STEP, IGES, DXF, etc.). Lightweight Collaboration formats are typically 3D formats derived from the native or neutral CAD model, which do not contain as much data as a native format or some neutral formats (e.g., JT, 3D PDF, 3Dxml, etc.).

According to Sun et. al. [2], the globalization of the modern manufacturing industry has lead to the need for tools that capture, represent, and exchange product development knowledge. The notion of the collaborative design process has emerged to support distributed and collaborative product development work teams, with suppliers and OEMs more tightly integrated. Current product development processes often include distributed workflows, complicated electronic interactions, and focused collaboration among the people involved. Historically, detail and assembly drawings were used to support these processes - they contained all of the necessary information to define, manufacture, and inspect the product $[3,5,6]$. However, drawings are also cumbersome in that they allow for ambiguity and incorrect interpretation of design intent, which can undermine the effectiveness of digital product definitions and collaborative design teams. Drawings also do not have the ability to provide robust derivations based on the background and technical literacy of the person viewing them, whereas model-based product definitions can be dynamically re-purposed based on the consumer of the data.

Another factor influencing the collaborative product development process is the nature of the digital environments themselves. Managing product data across continents using web-based computing architectures that must include appropriate security protocols is not a trivial matter. The design author produces a CAD model, rich with information. Yet that model is only consumable by a small portion of the organization in that form. However, due to corporate culture, regulatory compliance, and a number of other factors, most others in the organization are prohibited in their ability to modify this model. Moreover, their lack of technical ability and proper training will likely impede their ability to use this form of the model. 
As companies have difficulty in propagating the 'heavyweight' CAD model throughout the organization, the obvious need arises for a CAD-derivative format that is both dynamically manipulated and easily consumed. Hence the notion of making the model 'fit for purpose' by using alternate 3D formats to propagate data outside the native CAD software format. A fundamental requirement of a model-based product realization environment is that a fully-defined, fully-annotated 3D CAD model be the master source of product geometry definition. This allows for both human and machine consumption of model data within the larger collaboration framework. When people worked collaboratively in close physical proximity to each other, we trusted the data generated by them, because we trusted their personal judgment and expertise. In a global collaborative design and production environment, close physical proximity is not possible, thus requiring that we trust the process that generates the data as opposed to the person who generates the data.

Lightweight or collaboration formats were initially developed with visualization as the primary purpose. They could be used in a variety of scenarios such as design reviews, marketing, and product assembly on the shop floor. No special and expensive hardware or software was needed to view these formats. Over time these formats have evolved until they contain much more of the functionality found in native $\mathrm{CAD}$ system and have evolved for use by other scenarios such as manufacturing, coordinate measuring, and digital mock-up. As they have evolved, there has been extensive push from the user community for many of these formats to become recognized standards.

\section{The Product Development Process and the Digital Enterprise}

It is important to consider the system development process in concert with the collaborative product development environment. The concept of the V-model has emerged over the last three decades. It was introduced to simplify the complexity associated with system development. The V-model is applied across many industries to define a consistent approach to system design and product development. It begins with increasing degrees of product definition (concept to architecture to detail design) that leads to implementation (manufacture). From there the process traverses through component integration, test and verification and then system level integration, test and verification. Finally, culminating in the operation and fielding of a system that has been fully verified and validated against its defining requirements. But, how does model based collaboration and interoperability support and affect the V-model for complex system development shown in Figure 1 [7]?

The Digital Enterprise represents the evolution of processes through the use of digital models and information. There is the business information view (resources, finance, program management, etc.) as well as the technical view (design, manufacture, sustainability, etc.) of the digital enterprise. In the technical view, the Model Based Enterprise (MBE) includes the concept that shared digital product data is available, in a form that is accurate and appropriate, when and where it is needed. The ability to create data once and use it throughout the lifecycle, without the need to re-master data, is a key to the MBE.

Product models are transformed as the definition progresses throughout the product lifecycle - from concept to disposal. Within the model-based enterprise there are several important concepts. There is the Systems Engineering layer with information 


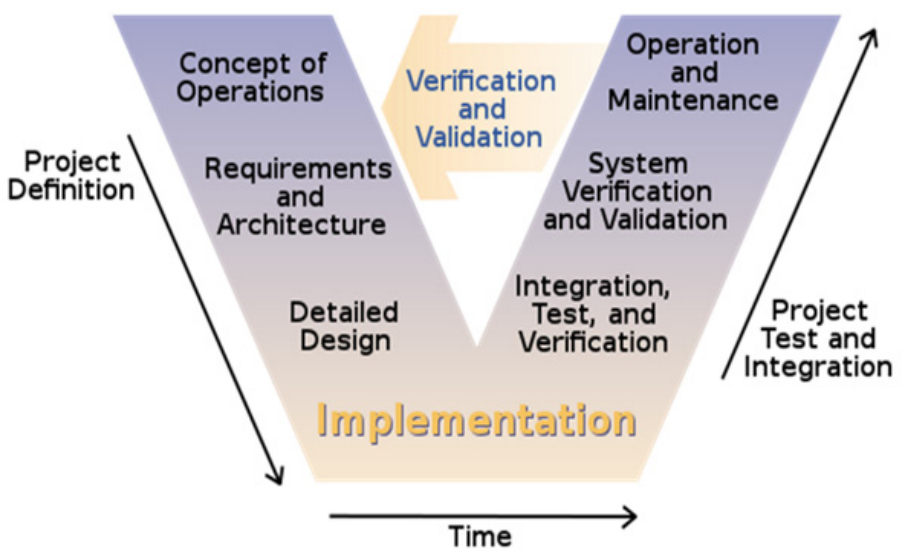

Fig. 1. V-model for Complex System Development

that spans the entire product life cycle. As the product model definition progresses through the stages of model based engineering, manufacturing, and sustainability, it is transformed from concept to retirement through these stages and is represented by product, process, and information models, as well as the collaborative visualization models. A robust MBE is inherently dependent upon the ease of data transformation, which is significantly improved through the collaborative capabilities of the modeling tools used to create the data and the standards used for data exchange. Appropriate application of the standards ensures that data flows seamlessly throughout the lifecycle and enables re-use of the data in formats that are most appropriate for collaboration and visualization throughout the lifecycle.

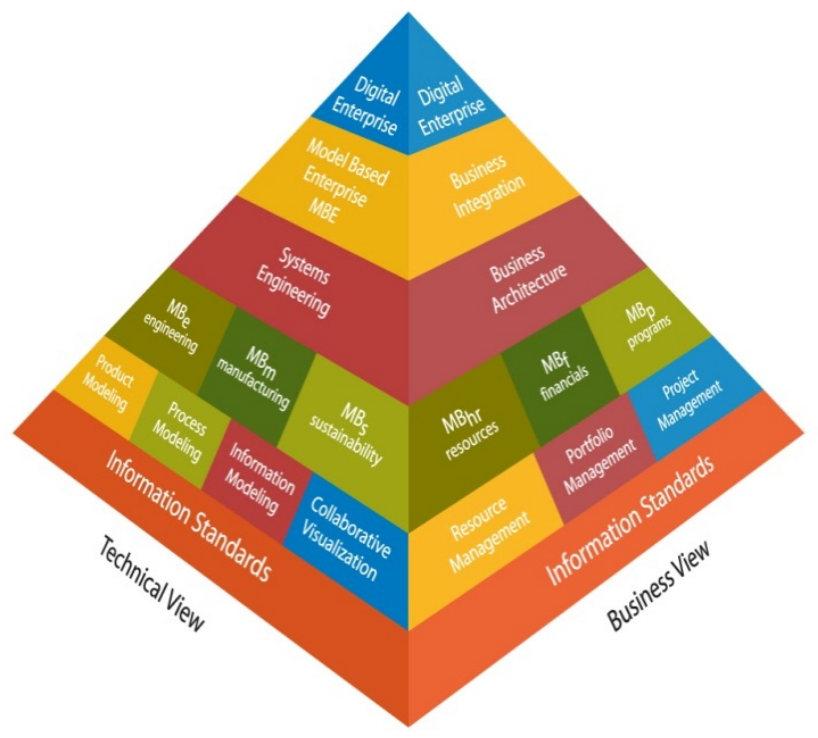

Fig. 2. Elements of a Model-based Enterprise 
Table 1 establishes the link between the Digital Enterprise pyramid and the Product Development V-diagram. Depending on where you are in an organization, and the type of organization you are in, there are other items involved in addition to the typical product lifecycle stages. Each actor has different collaborative, visualization and information needs in the development lifecycle. Business Portfolio Management is often maintained at a corporate or executive level within the organization. The Management Authorization Process is typically the responsibility of the program office (lower-level VP and directors). While the Product Data Lifecycle Stages (for reference from our outline) are usually directed by engineering managers, department managers, etc. Throughout all, as the product matures across the lifecycle, there exists the need to transform models and collaboratively share information for specific use cases. Usually the original model format is not conducive to the collaborative use case and often a lightweight visualization is more beneficial.

Table 1. The Link between the Digital Enterprise and Product Development

\begin{tabular}{llll}
\hline $\begin{array}{c}\text { Business Portfolio } \\
\text { Management }\end{array}$ & \multicolumn{1}{c}{$\begin{array}{c}\text { Program Management } \\
\text { Authorization Process }\end{array}$} & $\begin{array}{c}\text { Product Data Lifecycle } \\
\text { Stages }\end{array}$ & \multicolumn{1}{c}{ Use cases } \\
\hline $\begin{array}{l}\text { Strategic/financial } \\
\text { plan } \\
\text { Pursuit/Order capture }\end{array}$ & Validate options/trade-offs & Product specification & $\begin{array}{l}\text { Concept/requirements } \\
\text { capture } \\
\text { Proposal development }\end{array}$ \\
$\begin{array}{l}\text { Design \& } \\
\text { Development }\end{array}$ & Full-scale development & Design; Analysis & Design for X \\
Manufacturing & Manufacturing/Production & Manufacturing/Supply & $\begin{array}{l}\text { Quality; Supply chain; } \\
\text { Mfg. }\end{array}$ \\
Support \& Service & $\begin{array}{l}\text { Field Support; Stop } \\
\text { production }\end{array}$ & $\begin{array}{l}\text { Product support; Tech } \\
\text { pubs; TDPs }\end{array}$ & $\begin{array}{l}\text { Tech pubs; field } \\
\text { support }\end{array}$ \\
& Stop field support & $\begin{array}{l}\text { Long-term Archival \& } \\
\text { Retention }\end{array}$ & Data archival; disposal \\
\hline
\end{tabular}

Obviously the richest data format among the three we are examining here are the native CAD files. They have evolved over the past 30 years and are quite robust in nature. However, the downside of using these files for collaboration is multifaceted:

- $\quad$ They are unreadable by other CAD systems (without direct translators)

- Depending on the system, they may not be readable from one major release to the next

- If they are to be reused, they have to be "bumped up" or migrated on a regular basis from one release to the next

- They are typically rather large from a file size standpoint

Neutral files possess the greatest interoperability but obviously do not have the richness of the native CAD files. This is why the choice of which file format to use in a particular scenario is an important organizational decision. Lightweight collaboration formats have 
evolved as well and have become less "lightweight" as the functionality has increased. Another issue that has arisen as a result of this constant increase in functionality is that the collaboration formats need to be "bumped up" or migrated to later versions as the richness of the format is increased. In Figure 3 below, we compare the relative duration of time between when International Standards, Native CAD Formats, and Collaboration formats need to be migrated to later versions.

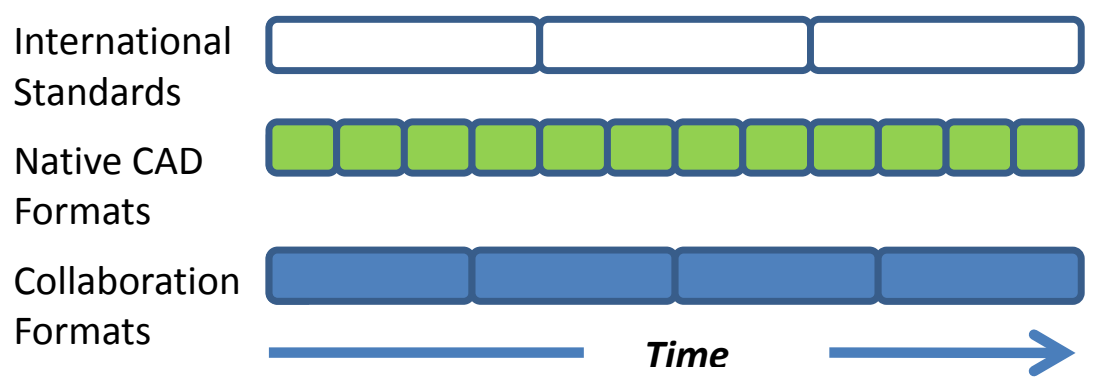

Fig. 3. The Progression of Product Data Formats

International Standards change relatively slowly - for example there have been three versions of STEP Application Protocol (AP) 203 and AP 214 commercially implemented in the past 17 years. For native CAD systems, major releases occur on an almost annual basis. Collaboration formats change less often than native CAD formats, but more often than international standards or neutral formats. Over a product's lifecycle, the information used to represent it and control it goes through a number of transformations and is integrated with other data as well. Over the course of its lifecycle, a product could be represented as a hand sketch, a CAD model, a set of NC tool paths, a lightweight visualization model, a set of assembly instructions, or a technical data package for service and repair. By the time a product is retired, the number of representations of it and the associated documentation is immense.

\section{The Nature of Collaborative Data}

In order to support collaboration between dispersed members of product teams in various stages of the product lifecycle, the digital product definition must be deep and wide in terms of the "richness" of its data. In most instances this means there is a fully detailed CAD model to support the communications process; however, it also means much more than that. The digital product definition also includes non-geometric metadata that is carried by the CAD file. The digital product definition must be dynamic and able to be reconfigured as necessary based on the needs of the consumer of the data. The CAD-derivative formats must also retain a high level of fidelity to the original CAD file (both geometric and non-geometric data) in order to be used successfully. High degrees of fidelity are particularly important in industry sectors (e.g., aerospace and defense, automotive, or pharmaceuticals) where regulatory compliance is necessary - not only for collaboration, but for archival purposes as 
well. The structure of the collaborative data must also be compact and stable with an ability to move across corporate networks easily, reliably, and securely.

In a model-based enterprise it is important that the digital product definition maintains stability, especially as the product definition evolves and transforms throughout the lifecycle. In order for the digital definition to fulfill the expectations of the system development (V-model) process, there must be a continuity of intent, whether the model is transformed for concept, physical/functional allocation, detailed design, collaborative visualization, analysis, manufacturing, service, support, or archival in the development lifecycle. No longer are concept, detail design, manufacture, test, service and support performed in a single location, nor are they typically done by a single corporation. The nature of today's supply chain is that of a supply network, and it is a driving component of the business environment and the world economy. There is a need to be able to collaborate across the different roles within the corporate organization and across the supply chain with other involved actors, all with a level of product representation fidelity that is acceptable for the actor in their role in the organization. When collaborating, the information needs to be of the appropriate richness, size, and most often contain only the intellectual property necessary to complete the particular use case.

Today's product development environment is highly collaborative and global. Figure 4 shows the typical stages in a product lifecycle. It is absolutely essential that information be shared by many different actors, in many different locations, and often simultaneously. Frequently, the information needed for different use cases requires product representations with significantly different formats or varying degrees of richness. The more we are able to create model data once and transform it seamlessly

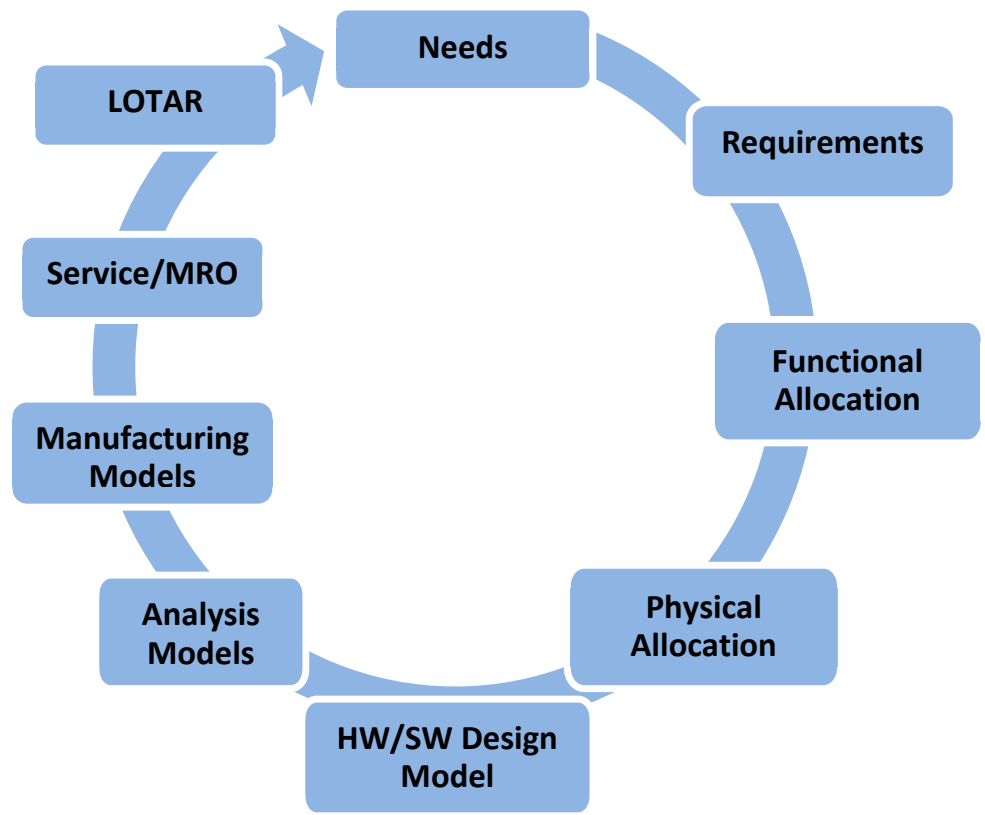

Fig. 4. Typical Stages of Product Lifecycle 
for other uses, and the less we re-master for different purposes, the more stable our product definition remains. With stability, companies can maintain efficiency, consistency, quality, and usefulness of the original design intent. To support this paradigm of model data re-use, it is typically necessary to use derivative model representations as not everyone in the product lifecycle needs the full mathematical definition provided by the native CAD model.

\section{Evaluation Framework for Collaborative Product Representations}

To address the variety of authors and consumers of data within the collaborative product lifecycle, four different types of product representations have evolved: native representations, derivative data models, derivative data formats, and derivative data packaging. In contemporary terms, this means technologies like STEP (data model), JT or 3DXML (format), or 3D PDF (package) that have been used as solutions for the challenges presented by various lifecycle workflows and the different data needs of consumers within the lifecycle. It is important to note that any specific strengths or weaknesses presented by a specific product representation are more often the result of the selection of that technology for a particular workflow than it is a specific technical deficiency. It has become common practice to use certain product representations in a manner inconsistent with its original development objectives. To address this issue, it is important to have a framework for evaluating the use and application of specific product representations.

Table 2. Evaluation Framework for Product Representation Selection

\begin{tabular}{|c|c|c|c|c|}
\hline & $\begin{array}{l}\text { Native format } \\
\text { (Authoring } \\
\text { system) }\end{array}$ & $\begin{array}{c}\text { Derivative data } \\
\text { model } \\
\text { (STEP) }\end{array}$ & $\begin{array}{l}\text { Derivative data } \\
\text { format } \\
\text { (JT or 3DXML) }\end{array}$ & $\begin{array}{c}\text { Derivative data } \\
\text { package } \\
\text { (3D PDF) }\end{array}$ \\
\hline Lifecycle Stages & \multirow{7}{*}{\multicolumn{4}{|c|}{$\begin{array}{ll}\text { - } & \text { Fidelity to functional requirement } \\
\text { - } & \text { Fidelity to physical allocation } \\
\text { - } & \text { Geometry representation (level of detail and ge } \\
\text { - } & \text { Metadata (semantics, materials, processes, etc. } \\
\text { - } & \text { Product structure } \\
\text { - } & \text { Openness } \\
\text { - } & \text { Extensibility } \\
\text { - } & \text { Accessibility } \\
\text { - } & \text { Security } \\
\text { - } & \text { Portability } \\
\text { - } & \text { Long-term stability of product representation }\end{array}$}} \\
\hline Requirements capture & & & & \\
\hline Proposal development & & & & \\
\hline Product definition & & & & \\
\hline Supply chain integration & & & & \\
\hline Field Support & & & & \\
\hline Data archival & & & & \\
\hline
\end{tabular}


For the purpose of this paper, instead of discussing workflows that tend to be popular among the user communities such as Detailed Design, Design Collaboration, and Product Manufacturing, we focused efforts on workflows not normally considered such as Requirements Capture, Product Definition and Development, Field Support, and Data Archival. For the purposes of our workflows, we considered what inputs typically are required, what primary process they are driving, and what downstream process is being fed by the information. In light of this process, a framework for evaluating product representations was developed, with specific criteria to guide the review. The criteria in Table 2 need to be applied in the context of intended inputs and outcomes by the authors and consumers of the data in each respective workflow.

For Requirements Capture, inputs are typically Business Strategy, Customer Requirements, information that has been gleaned by Marketing, and new Technology that has been recently developed or is in the process of being developed. The inputs for Requirements Capture come in a variety of formats such as word processing documents, spread sheets, and CAD models. This information goes to the Systems Engineering organization and is used for defining system requirements, and validating initial requirements. The information generated by the Systems Engineering organization is fed to Conceptual Design.

For proposal development, input data is provided by the Design Engineering, Manufacturing Engineering, Finance, and Marketing organizations. The information they provide is in a variety of formats, including derivative data formats, word processing documents, and spread sheets. This data is provided to the Program Office. They in turn use the information for the development of proposals, pursuit and order capture, and early or initial requirements validation. The output of this is a proposal to the customer.

In the product definition workflow, input data is provided by program management, systems engineering, manufacturing, customers, as well as several other entities. The information they provide is typically contained in text-based documents, requirements document, systems modeling language, or geometric representations in the form of previous designs. Inside design engineering, activities include concept design, trade studies, detail design, and analysis. The focus on interoperability between authoring systems both within functions and between functions (MCAD-toMCAD, MCAD-to-CAE, MCAD-to-ECAD, etc.) requires the use of a flexible product representation. The output of this process is a data set that can be consumed readily by manufacturing to generate product structures, model-based work instructions, tooling, and computer-aided manufacturing code.

In Supply Chain Integration input data from the Design Engineering, Quality, Manufacturing, and Program Management organizations is provided to Materials Management. This information typically takes the form of neutral files, native CAD files, derivative data formats, word processing documents, spread sheets, and "electronic" schedules. The Materials Management organization uses this information to perform make/buy decisions, decide on the level of collaboration with suppliers, and perform procurement. The outputs from the Materials Management organization take a number of forms, such as requests for information, requests for proposal, and contracts and purchase orders. 
For Field Support, the Engineering and Manufacturing organizations and the customer provide the data to the Field Support organization. The information is provided in a number of formats such as derivative data formats, native CAD models, word processing documents, and spread sheets. The Field Support organization uses this information to create technical publications, provide logistics support, make decisions for provisioning, and analyzing field data for future decisions. Much of the output of the Field Support function goes to the Service Centers where the products are maintained and updated.

For Data Archival, Design Engineering, Manufacturing, and Information Management all provide input to the Data Control organization. This information is in a variety of formats including native CAD models, derivate data formats, $\mathrm{NC}$ tool path data, PLM/PDM information in both neutral and native formats, various document formats, spread sheets, and databases. The Data Control organization performs change management; they convert and validate the data, and finally archive the data. Nearly constant data migration is also performed depending on the information format.

\section{Summary}

One of the fundamental tenets of product lifecycle management and a model-based enterprise is the movement of information throughout the enterprise in a way than enables effective decision making through the use of a high-fidelity digital product representation. In order for the various stages of the product lifecycle shown in Figure 4 to be effective, they must have proper inputs and outputs that retain the integrity of the digital product definition. The nature of the digital product definition is more than just a CAD model, more than just geometry. It includes non-geometric attributes that drive downstream processes because they are made fit for machine consumption rather than human consumption. Machine-to-machine communication is necessary in many facets of the product lifecycle as a human in the loop can be too slow and (potentially) inaccurate. Yet, a fully-integrated product lifecycle only works if the geometric and non-geometric definitions of the product remain intact during translation and the dynamic re-purposing of the model-based product definition. It is critical that the authors and consumers in the lifecycle conduct due diligence to evaluate specific product representations within each workflow. Using the criteria mentioned in Table 2 will aid organizations in selecting an appropriate product representation.

The product lifecycle and its accompanying processes and workflows have become increasingly complex as the use of digital data has continued to supplant paper-based processes. The authoring tools used to create such data (e.g., CAD systems, spreadsheet tools, analysis tools, visualization platforms, etc.) write their output in proprietary formats which vary across the product lifecycle based on the specific workflow and the consumer characteristics involved. Too often companies find themselves in the predicament of choosing between various product representations native (proprietary) and derivative - when the decision is not really a choice, but a selection of complementary technologies. The use of particular product representations does not (and should not) need to be mutually exclusive. 
The product representations used across the lifecycle will vary with respect to the desired functionality at each stage. The exclusive use of one class of representation over another can limit flexibility. Use of native CAD formats in the design or analysis stage of the lifecycle is likely; however, the use of those same types of files in the purchasing or technical publications stages of the lifecycle simply do not make sense. A native file would be too large in size, have too much detail, and would likely not be able to be opened by the tools that people in those positions would use. Derivative data formats can promote high fidelity communications between design and manufacturing or design and supply chain, but tend to be too large in size for publications and are usually not editable enough to be dynamically culled for levels of detail if the neutral file was not saved with an appropriate level of detail for a specific stage in the lifecycle. A lightweight collaborative file could be used for mobile applications, obtaining supplier quotes, or publications, but likely not for machining in a CNC application. Combining native, neutral and collaborative formats provide a richer solution across organizations and the lifecycle. The key to this selection process is the application of the selection criteria for each product representation based on the inputs and outputs of the respective workflows.

\section{References}

1. Hartman, N.W., Lim, A.: Examining neutral formats for visualization and data exchange. In: International Conference on Engineering and Technology, IAJC/IJME 2008, Nashville, TN, United States, p. 14 (2008)

2. Sun, D.W., Xiong, X.H., Ruan, L.W., Liu, Z.J., Zhao, J.M., Wong, Y.S.: Workflow-driven collaborative session management in product lifecycle management via Internet. In: Proceedings 2004 IEEE International Engineering Management Conference, October 18-21, vol. 3, pp. 1146-1150 (2004), doi:10.1109/IEMC.2004.1408872

3. Quintana, V., Rivest, L., Pellerin, R., Kheddouci, F.: Re-engineering the Engineering Change Management process for a drawing-less environment. Computers in Industry (63), 79-90 (2012)

4. Boucher, M.: Working with multi-CAD: Overcome the engineering collaboration bottleneck (December 2010),

http: / / aberdeen. com/aberdeen-library/6752 / RA-designengineering-collaboration. aspx (retrieved from February 3, 2012)

5. Quintana, V., Rivest, L., Pellerin, R., Venne, F., Kheddouci, F.: Will model-based definition replace engineering drawings throughout the product lifecycle? A global perspective from aerospace industry. Computers in Industry 61(5), 497-508 (2010)

6. Versprille, K.: Model-based Definition for the Masses. Collaborative Product Development Associates.,

http://www.righthemisphere.com/_base/static/img/

whitepapers/2_cpda_rh_wp.pdf

7. Clarus Concept of Operations. Publication No. FHWA-JPO-05-072, Federal Highway Administration, FHWA (2005) 